\title{
Test Methods to Evaluate the Effect of Interface Bond Conditions on Top-Down and Reflective Cracking
}

\author{
Yu Chen*, George Lopp, and Reynaldo Roque \\ University of Florida, Civil \& Coastal Engineering, Gainesville, Florida, USA \\ E-mail: ychen@ufl.edu*
}

\begin{abstract}
A composite specimen interface-cracking (CSIC) test was developed to evaluate the effect of the characteristics of the interface between asphalt layers on resistance to topdown and reflective cracking. The system involves repeated tensile loading and monitoring of the rate of damage development (reduction in stiffness) on composite specimens specifically designed for this purpose. Top-down cracking tests were performed on opengraded friction course (OGFC) mixtures placed on dense-graded mixtures using both a conventional tack coat and a polymer-modified asphalt emulsion (PMAE) at a higher application rate. Reflective cracking tests were performed on dense-graded mixtures. Results clearly indicated that use of polymer-modified bonding material significantly reduced the rate of damage development for both top-down and reflective cracking. The testing and interpretation methods developed can be used to optimize bonding materials and application rates for enhanced cracking performance. The methods may also provide a suitable specification test for bonded systems.
\end{abstract}

Keywords: Interface, bond, asphalt, cracking, fracture.

ENGINEERING JOURNAL Volume 16 Issue 4

Received 7 January 2012

Accepted 24 February 2012

Published 1 July 2012

Online at http://www.engj.org/

DOI:10.4186/ej.2012.16.4.91 


\section{Background}

OGFC has been widely used in the United States to improve road safety under wet conditions because of its much higher percentage of air voids, in the range of $15 \%$ to $25 \%$, as compared with dense graded mixture [1]. However, most of the OGFC research work done so far has been function related and most pavement design guidelines consider the OGFC as a wearing surface layer with no structural value. For pavements with OGFC, it was recognized that the OGFC mixture may be the 'first front' in resisting topdown cracking. However, due to its inherently high air voids and accelerated aging caused by direct exposure to UV radiation and high surface temperatures, OGFC mixtures have been reported to have considerably lower values of fracture energy density and dissipated creep strain energy to failure than dense graded asphalt mixtures [2]. Analysis of findings from pavement field sections in Florida has clearly indicated that the quality of the OGFC and the characteristics of the interface between OGFC and the structural mixture affect top-down cracking performance. In addition, recent laboratory work at the University of Florida [3] suggests that cracks that develop either in the OGFC or the hot mix asphalt structural layer can be effectively arrested and/or deterred by improving the characteristics of the bonded interface between the OGFC and the underlying structural mixture. In addition, pavement stress analyses have clearly indicated that debonding at the interface between asphalt layers results in stresses that are conducive to surface cracking $[4,5,6]$. Therefore, a reliable testing system and data interpretation method are needed for evaluation of the effects of characteristics of the interface between asphalt layers on resistance to top-down and reflective cracking.

\section{Objectives}

The objectives of this study are as follows:

- Evaluate a new testing and data interpretation system developed to assess the effect of interface characteristics on pavement cracking performance.

- Present specimen preparation, testing and data interpretation methods.

- Evaluate the effect of interface bond conditions on top-down and reflective cracking.

\section{Scope}

This study primarily focused on the presentation of newly developed testing, evaluation, and data interpretation method that allows for the characterisation of the effect of bonded interfaces on top-down and reflective cracking resistance. For top-down cracking, composite specimen interface cracking (CSIC) tests were performed on OGFC mixtures placed on dense graded mixture using both conventional tack coat and polymer-modified asphalt emulsion (PMAE). For reflective cracking, CSIC tests were performed on dense graded mixture placed on dense graded mixture using both conventional tack coat and PMAE. For both tests, one application rate was examined for each interface type, as this study was not meant to optimize application rate. All tests were conducted at one temperature $\left(10^{\circ} \mathrm{C}\right)$, which has been determined in prior fracture research at the University of Florida to correlate well with cracking performance of pavements in the field.

\section{Test and Data Interpretation Methods}

\subsection{Overview}

Currently, tests available for pavement layer interface evaluation mainly focus on interface shear resistance. In addition, most existing tests are performed in monotonic mode, which is not representative of the pavement interface loading conditions under traffic [7]. Also, tests used to evaluate cracking resistance of interlayer materials require large specimens, which are relatively difficult to fabricate in the laboratory and more difficult to obtain from the field [8-12]. Tests commonly used to the determine the tensile properties of asphalt mixture; e.g., Superpave Indirect Tensile Test [13] and uniaxial tension test [14, 15] are designed to evaluate single asphalt mixture; they are not suitable for evaluating composite specimens normally composed of two types of asphalt mixtures with an interface in between. Therefore a new testing system, the CSIC test was designed to propagate a crack from one material layer through the interface to the other 
material layer(s), using easily obtained specimens, i.e. Superpave gyratory compacted specimens or cored field specimens.

\subsection{Specimen Preparation and Test Method}

Test specimens can be prepared from either Superpave gyratory compacted specimens or cored field specimens. The laboratory preparation process involves compaction, cutting, gluing and grooving as shown in Fig. 1. The test specimen consisted of two separately prepared composite specimens bonded together at the pavement surface for top-down cracking and at the bottom of the structural layer for reflective cracking. The top-down cracking specimens were composed of a $25 \mathrm{~mm}$ thick OGFC mixture compacted on dense graded mixture; whereas the reflective cracking specimens were composed of a $19 \mathrm{~mm}$ dense graded structural layer on a dense graded overlay. For both specimen types, a $19.05 \mathrm{~mm}$ diameter stress concentrator is cored at the specimen center. The final specimen was trimmed to $38 \mathrm{~mm}$ depth from the original $150 \mathrm{~mm}$ diameter composite specimen. The specimen's curved ends were reinforced with carbon fiber to eliminate a potential bending failure.
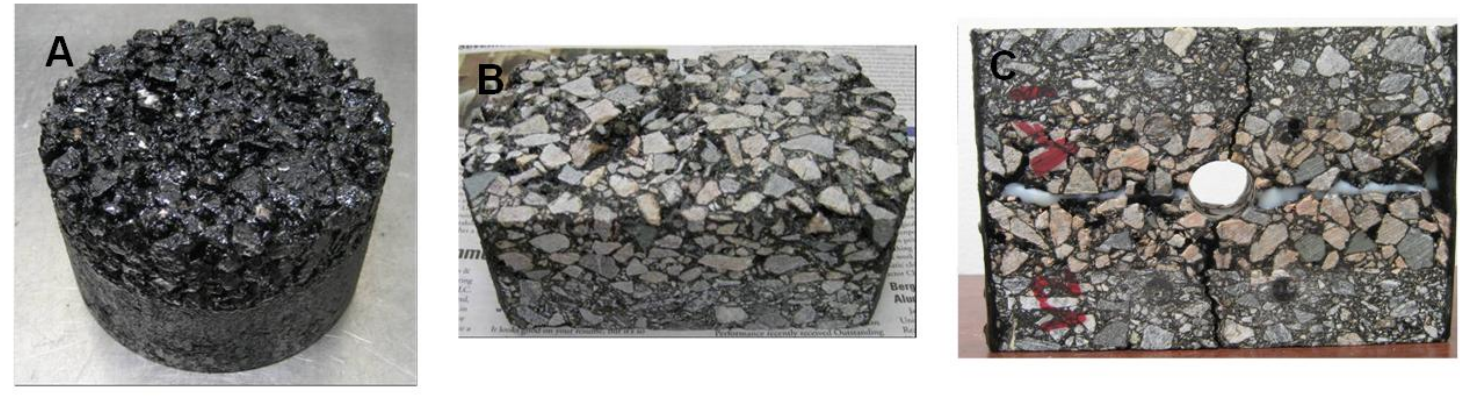

Fig. 1. Composite specimen preparation process.

The CSIC test was performed by applying a repeated haversine load for 0.1 second followed by a 0.9 second rest period by way of two split cylinder yokes inserted in the hole in the center of the specimen (see Fig. 2). The radius of the two yokes was $9.5 \mathrm{~mm}$, matching the radius of the stress concentrator in order to ensure uniform contact and to properly distribute the load. A seating load of $44 \mathrm{~N}$ was applied to ensure the specimen always in tension and that contact was never lost between the yokes and specimen. Four extensometers, two on each side of the specimen, were centrally mounted on gage points attached to the specimens at a distance of $19.1 \mathrm{~mm}$ from the composite specimen symmetrical plane. A plan view sketch of a specimen depicting load and measurement system is shown in Fig. 2 (dimensions in parentheses correspond to reflective cracking specimens).

\subsection{Data Collection and Interpretation Method}

Extensometer data was acquired at a rate of 5 samples per second and plotted to allow the operator to observe for abrupt changes in deformation as an indication of local damage evolution. If a sudden change occurred, or whenever desired, the operator recorded a burst of data for 6 consecutive loading cycles at a rate of 500 data points per second, which allowed for calculation of the specimen's total recoverable deformation (see Fig. 3).

The total recoverable deformation, which is inversely related to the specimen's stiffness, was calculated to facilitate comparison of the specimen's behavior and performance throughout the test. The specimen stiffness was calculated from the average of the total recoverable deformation of the four extensometers, and the average applied load for the six load cycles. Do not include headers, footers, or page numbers other than those already set in this manuscript. Note that the headers, footers or page numbers are different for the first page and the rest of the pages. Actual page numbers and other running heads will be modified when publications are assembled. 
(a)

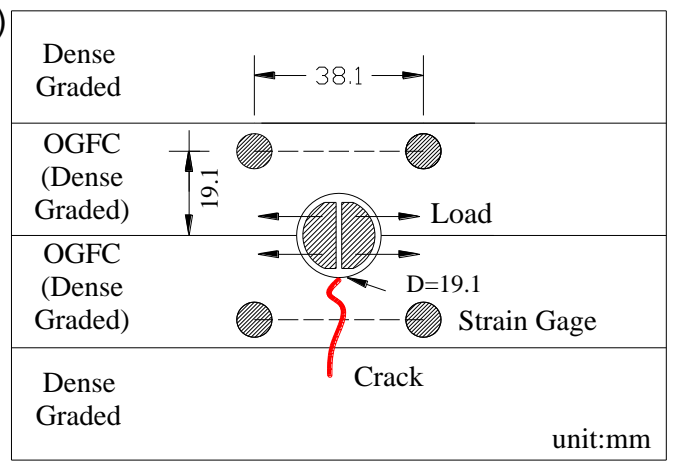

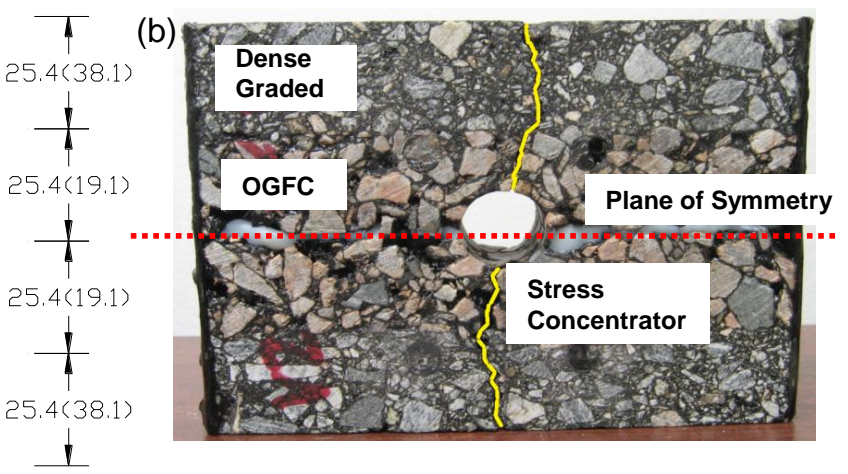

Fig. 2. (a) Specimen loading and measurement system and (b) cracking mechanism.

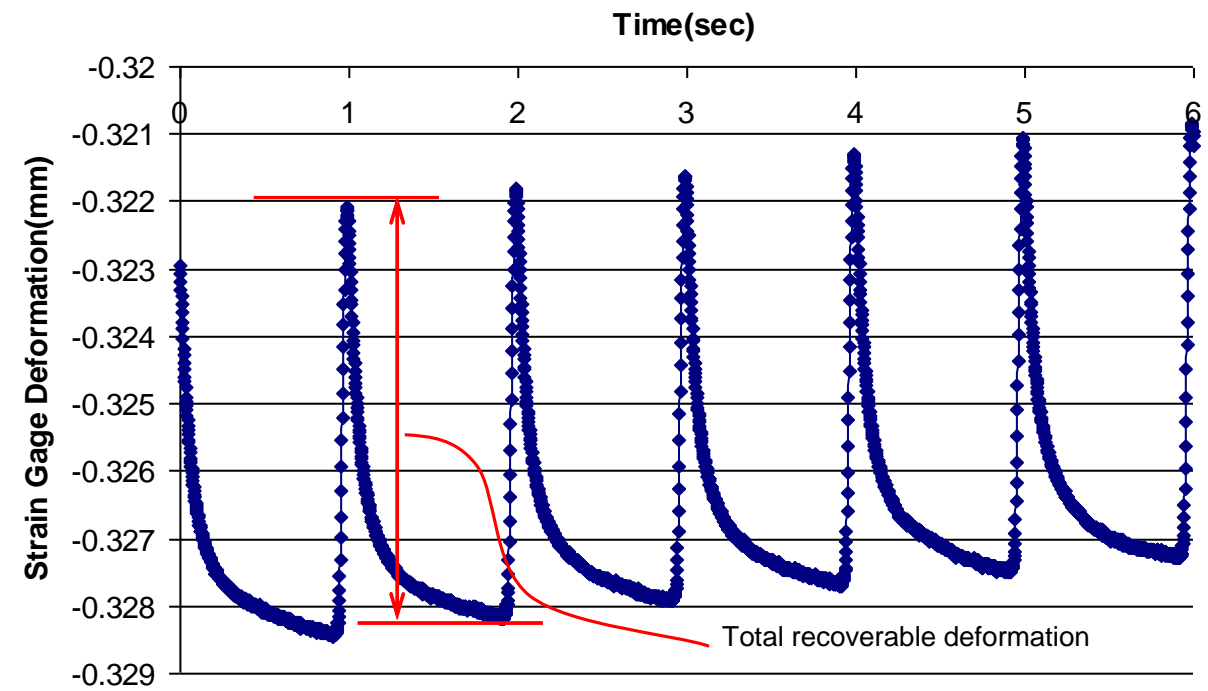

Fig. 3. Total recoverable deformation.

The total number of load cycles required to break the composite specimen was used as a straightforward cracking resistance comparison parameter for specimens with different interface conditions subjected to the same loading conditions. However, this parameter provided only the fracture resistance of the whole specimen without any information regarding the damage evolution in the specimen.

It has been well recognized that damage induced in the specimen can be measured by the specimen's stiffness reduction. As indicated earlier, the total recoverable deformation measurement is inversely related to the stiffness, so the change in total recoverable deformation can be used to monitor damage. A typical total recoverable deformation versus time plot is shown in Fig. 4. As shown in this figure, the total recoverable deformation versus time curve can be divided into three stages: the initial stage, which is known to involve changes in temperature and local damage adjacent to the loading yokes; the second stage, which involves steady-state damage; and the final stage, when the crack propagates rapidly and the specimen breaks. The damage rate was defined as the slope of the steady state response portion of total recoverable deformation progression curve as shown by the line in Fig. 4. 


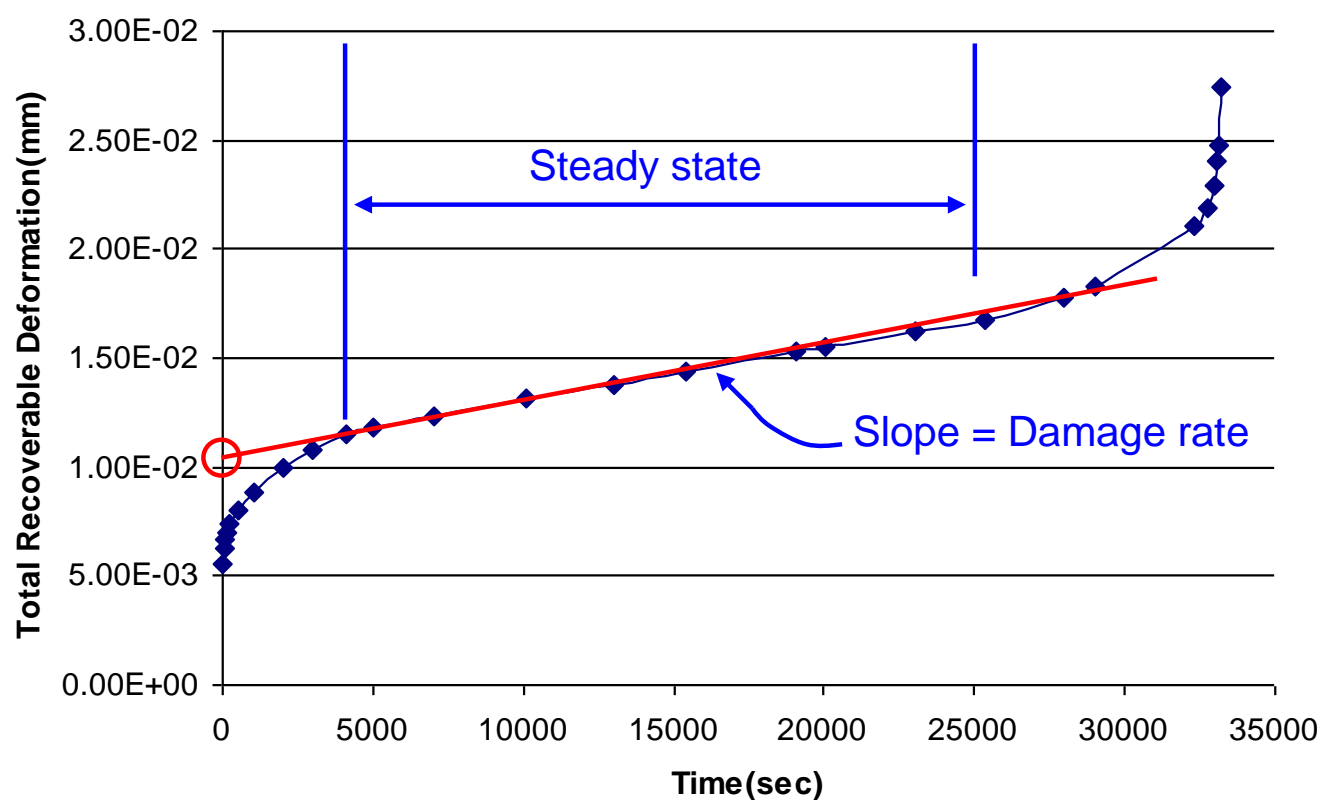

Fig. 4. Typical total recoverable deformation and damage rate.

\section{Test Results}

\subsection{Top-down Cracking}

The composite specimens used for this evaluation were prepared with OGFC and dense graded mixture designs commonly used in Florida. A conventional tack coat and PMAE (Novabond) from Road Science, LLC, were applied on the dense-graded specimen surface at an application rate of $0.204 \mathrm{l} / \mathrm{m}^{2}$ and 1.358 $1 / \mathrm{m}^{2}$, respectively. Based on finite element model (FEM) results and prior research experience with these materials it was determined that a peak load of $2535 \mathrm{~N}$ was appropriate. Three replicate specimens were prepared and tested for both the conventional tack coat interface and the Novabond interface.

Figures 5 and 6, which show test results for top-down cracking specimens in terms of number of cycles to failure and damage rate, clearly indicate that specimens with the Novabond interface outperformed the specimens with conventional tack coat in terms of cracking resistance. It appears that the relatively thick nature of the PMAE at the interface, along with the higher strain tolerance and excellent bond characteristics of the PMAE, results in an interface that can more effectively dissipate tensile stresses and provide greater resistance to damage. It is well known that the PMAE migrates upward into the high air void content OGFC, thereby filling voids in the aggregate and creating an interface of high cohesion. The CSIC successfully distinguished the expected difference in cracking resistance between these two interfaces.

\subsection{Reflective Cracking}

Three replicate specimens for each of the two types of interfaces, $0.453 \mathrm{l} / \mathrm{m}^{2}$ diluted conventional tack coat and $0.905 \mathrm{l} / \mathrm{m}^{2}$ Novabond, were prepared by Road Science, LLC for reflective cracking tests. The completed specimen, which involved the introduction of a Teflon spacer to represent an existing crack, which more effectively concentrated stress at the interface, is shown in Fig. 7.

Figures 8 and 9, which show test results for top-down cracking specimens in terms of number of cycles to failure and damage rate, clearly indicate that specimens with the Novabond interface outperformed the specimens with conventional tack coat in terms of resistance to reflective cracking. Tests were performed at two load levels (1912 N and $2312 \mathrm{~N}$ ), which correspond to low stress and high stress tests in Figs. 8 and 9. The enhanced performance of the Novabond interface was observed for both stress levels. 


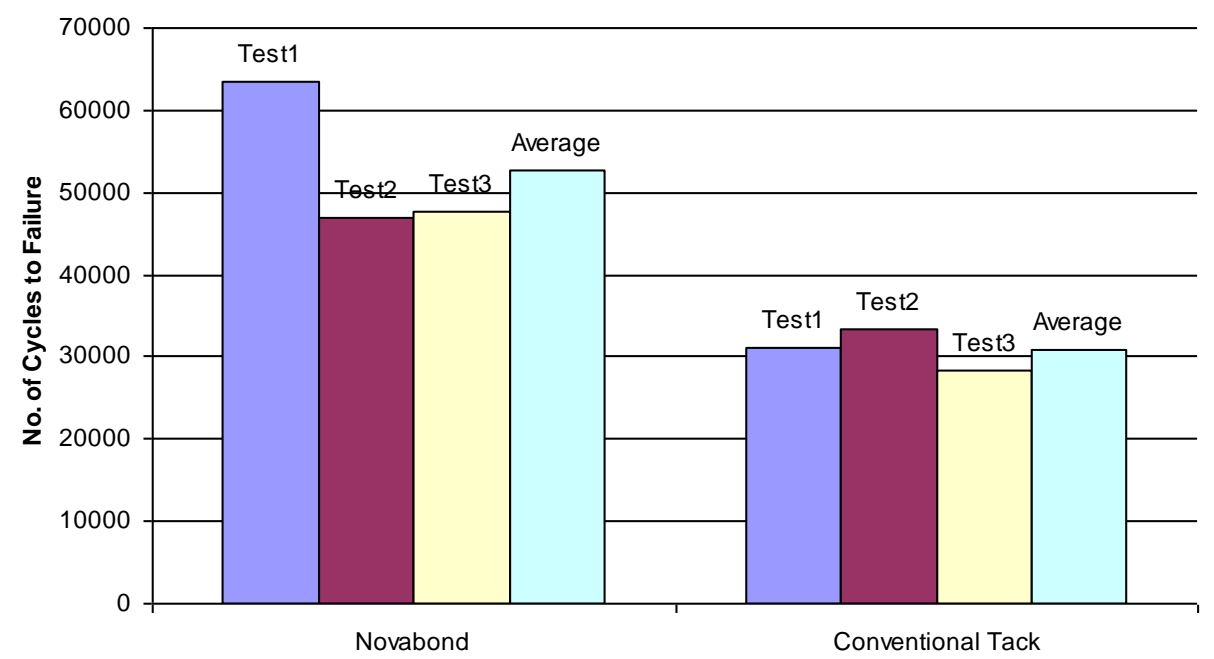

Fig. 5. Total number of cycles to failure for Novabond and conventional tack.

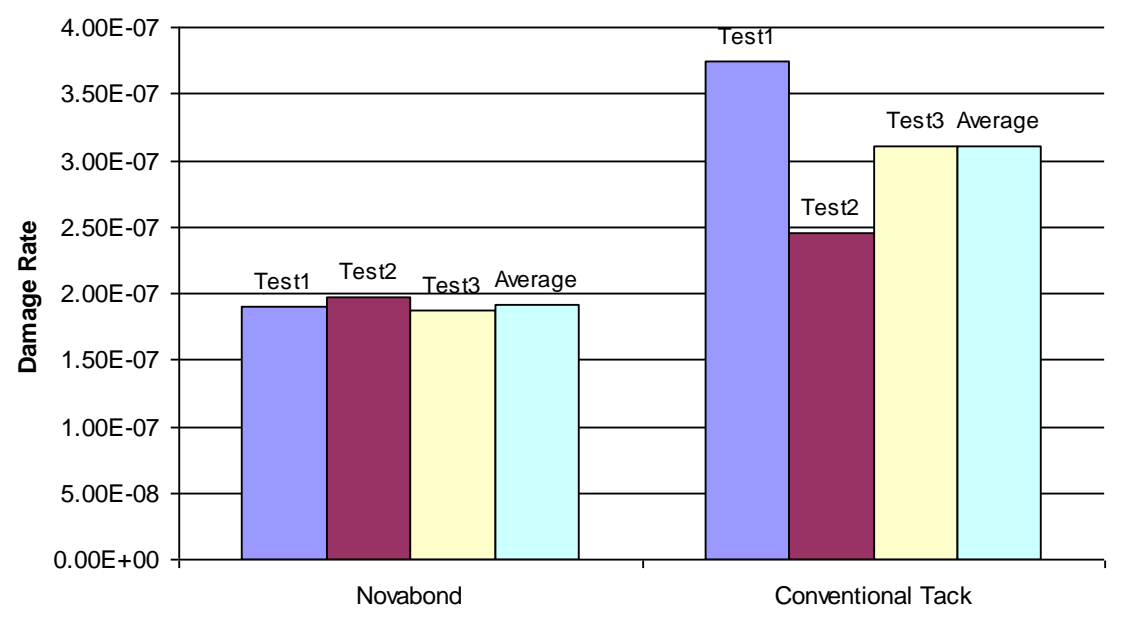

Fig. 6. Damage rates of Novabond and conventional tack.

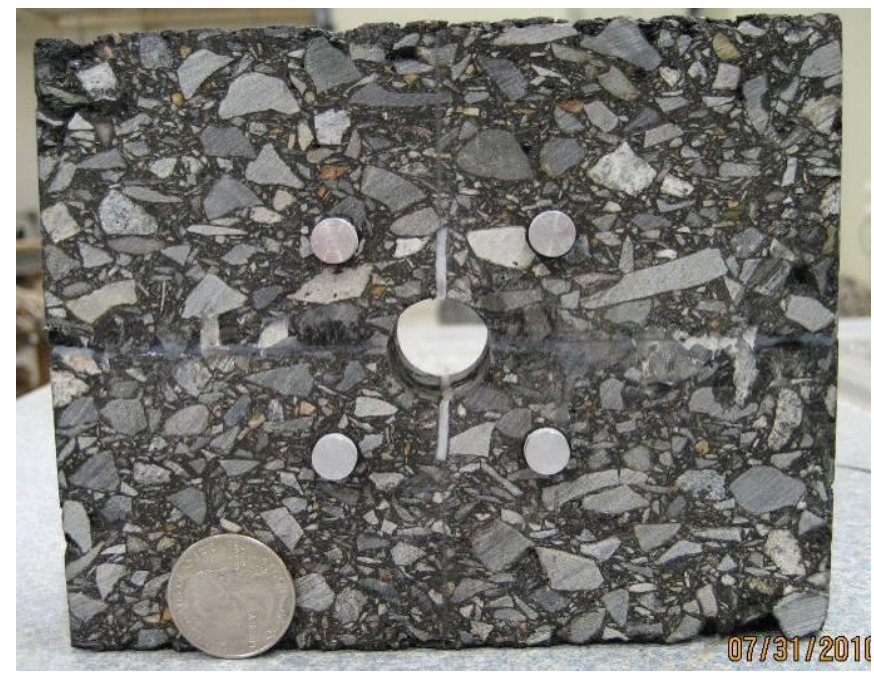

Fig. 7. Composite specimen with Teflon spacer. 


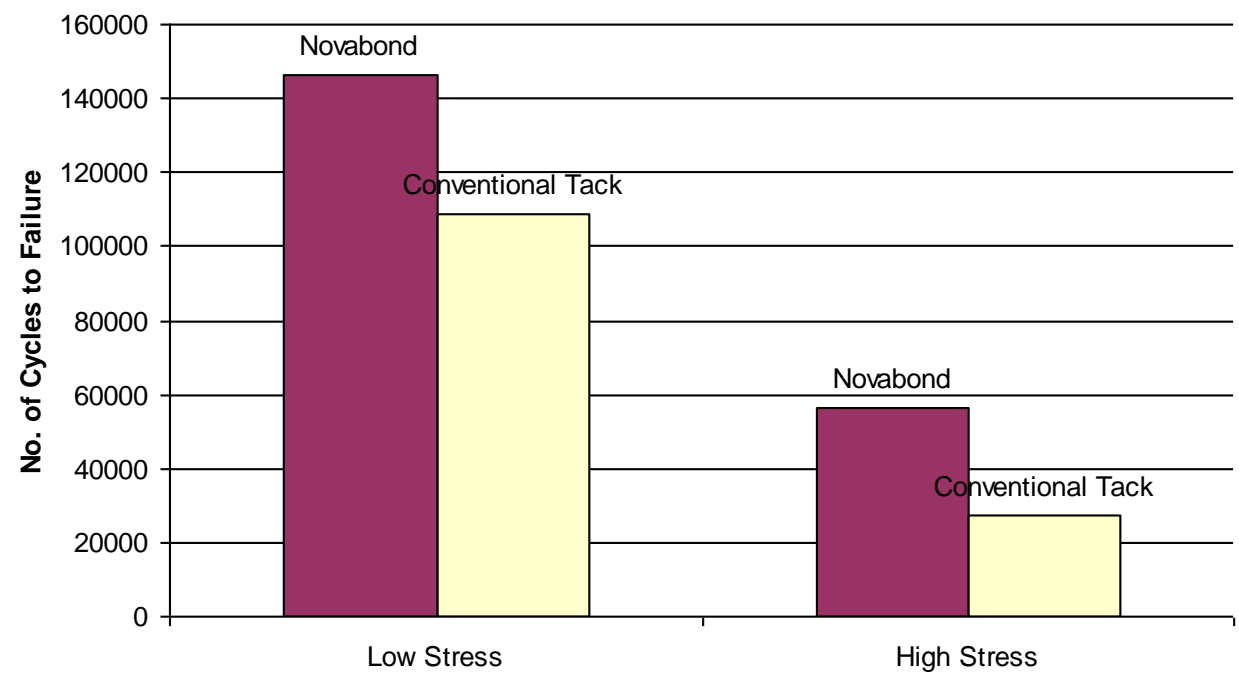

Fig. 8. Total number of cycles to failure for Novabond and diluted conventional tack.

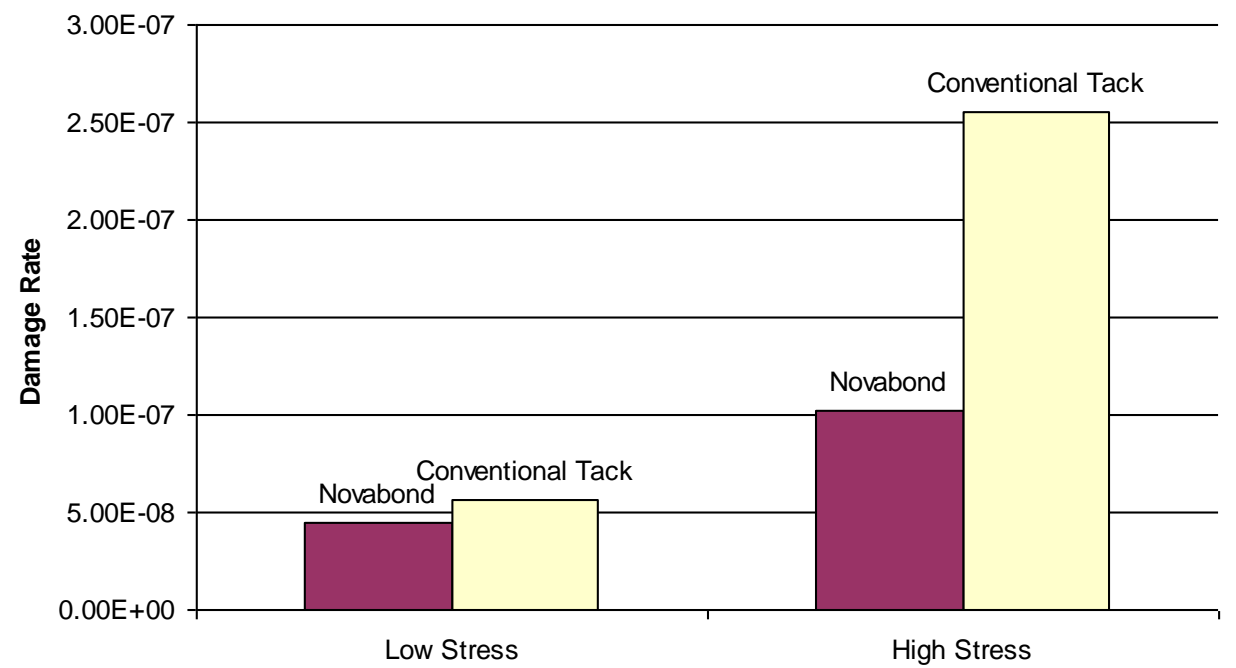

Fig. 9. Damage rates of Novabond and diluted conventional tack.

It appears that the Novabond PMAE was able to effectively dissipate tensile stresses even between two dense-graded mixtures. It should be noted that this benefit was observed in laboratory prepared specimens, for which it is possible for some of the PMAE to squeeze out from the interface during compaction (as compared to the field, where the PMAE has nowhere to go).

\section{Conclusion}

In this study, it was shown that the newly developed CSIC test successfully distinguished effects of pavement layer interface characteristics on both top-down and reflective cracking performance. The following conclusions can be drawn from this study:

- This newly introduced test method can serve as tool for evaluating the cracking performance of interface materials on pavements.

- The total number of cycles to failure, and damage rate are two effective measurements of cracking performance for composite specimens with different interface conditions.

- Novabond PMAE increased both top-down and reflective cracking performance of composite specimens by increasing the cracking resistance of materials near the interface and by dissipating the stresses at or along the interface. 


\section{References}

[1] P. S. Kandhal and R. B. Mallick, "Open graded friction course: state of the practice," National Center for Asphalt Technology, Auburn, AL, NCAT Report 98-7, 1998.

[2] C. Koh, "Tensile properties of open graded friction course (OFGC) mixture to evaluate top-down cracking performance," Ph.D. dissertation, University of Florida, Gainesville, FL, 2009.

[3] B. Birgisson, R. Roque, A. Varadhan, T. Thai, and L. Jaiswal, "Evaluation of thick open graded and bonded friction courses for Florida," Florida Department of Transportation, University of Florida, Gainesville, FL, Final Rep., 2006.

[4] M. Y. Shahin, K. Kirchner, E. W. Blackmon, and H. Tomita, "Effect of layer slippage on performance of asphalt-concrete pavements," Transportation Research Record, no. 1095, pp. 79-85, 1986.

[5] M. Ameri-Gaznon and D. Little, "Octahedral shear stress analyses on an ACP overlay on a rigid base," in Proceedings of the Association of Asphalt Paving Technologists, 1990, vol. 59, pp. 443-479.

[6] S. Gomba, J. Liddle, and Y. A. Mehta, "Evaluation of interlayer bonding in hot mix asphalt pavements," The International Journal of Pavements, vol. 4, no. 1 and 2, pp. 13-24, 2005.

[7] C. Raab, M. N. Partl, and A. E. H. O. A. El Halim, "Evaluation of interlayer shear bond devices for asphalt pavements," Baltic J Road Bridge Eng, vol. 4, no. 4, 176-195, 2009.

[8] M. T. Mukhta, and B. J. Dempsey, "Interlayer stress absorbing composite (ISAC) for mitigating reflection cracking in asphalt concrete overlays," Illinois Cooperative Highway Research Program, Final Report Project IHR-533, 1996.

[9] K. W. Kim, Y. S. Doh, and S. Lim, "Mode I reflection cracking resistance of strengthened asphalt concretes," Construction and Building Materials, vol. 13, no. 5, pp. 243-251, 1999.

[10] S. F. Brown, N. H. Thom, and P. J. Sanders, "A study of grid reinforced asphalt to combat reflection cracking," in Proceedings of the Association of Asphalt Paving Technologists, 2001, vol. 70, pp. 543-571.

[11] F. Zhou and T. Scullion, "Overlay tester: A rapid performance related crack resistance test," Texas Transportation Institute, Report No. FHWA/TX-05/0-4467-2, 2004.

[12] A. Khodaii, S. Fallah, and F. M. Nejad, "Effects of geosynthetics on reduction of reflection cracking in asphalt overlays," Geotextiles and Geomembranes, vol. 27, pp. 1-8, 2009.

[13] R. Roque, W. G. Buttlar, B. E. Ruth, M. Tia, S. W. Dickison, and B. Reid, "Evaluation of SHRP indirect tension tester to mitigate cracking in asphalt pavements and overlays," Florida Department of Transportation, Tallahassee, Final Rep., Aug. 1997.

[14] P. Bolzan and G. Huber, "Direct tension experiments," National Research Council, Washington, DC, Strategic Highway Research Program-A-64 1, 1993.

[15] S. W. Park, Y. R. Kim, and R. A. Schapery, "A viscoelastic continuum damage model and its application to uniaxial behavior of asphalt concrete," Mechanics of Materials, vol. 24, no. 4, pp. 241-255, 1996. 